\title{
Evolution of mitochondrial genomes in three closely-related Armillaria species
}

\author{
A. Kolesnikova ${ }^{1}$, Yu. Putintseva ${ }^{1}$, S. Jain ${ }^{1}$, N. Oreshkova ${ }^{1,2}$, I. Pavlov $^{1,2}$, V. Sharov ${ }^{1}$, \\ D. Kuzmin ${ }^{1}$, S. Makolov ${ }^{1}$, K. Krutovsky ${ }^{1,3,4,5 *}$ \\ ${ }^{1}$ Siberian Federal University, Krasnoyarsk, Russia \\ ${ }^{2}$ V.N. Sukachev Institute of Forest SB RAS, Krasnoyarsk, Russia \\ ${ }^{3}$ Georg-August University of Göttingen, Göttingen, Germany \\ ${ }^{4}$ N.I. Vavilov Institute of General Genetics RAS, Moscow, Russia \\ ${ }^{5}$ Texas A\&M University, College Station, USA \\ *e-mail:konstantin.krutovsky@forst.uni-goettingen.de
}

Key words: mitochondrial genome, mitochondrial evolution, homing endonuclease genes, plasmid DNA

Motivation and Aim: Comparative analysis of mitochondrial genomes of closely related organisms allows obtaining new information about their phylogenetic and evolutionary relationships. Plant and fungi mitochondrial genomes are especially interesting due to their large mtDNA size.

Methods and Algorithms: In this study we analyzed mitochondrial genomes of Armillaria borealis (116443 bp) and A. sinapina (103563 bp) sequenced and annotated in Laboratory of Forest Genomics of the Siberian Federal University. The complete sequence of the $A$. solidipes (122167 bp) mitochondrial genome was retrieved for annotation and comparative analysis as a single scaffold from the JGI Genome portal. Genome rearrangements were detected using the MAUVE 2.0 program. Duplicated sequences were identified by local BLASTn searches of mtDNAs against themselves with a cut-off e-value of $10^{-3}$. Intronic nucleotide sequences of three species were compared with each other and NCBI GenBank database using BLAST.

Results: Despite conserved gene content, there were significant genome rearrangements in a region between $r p s 3$ and atp 9 genes. We found gene duplications in all species. There was an $87 \mathrm{bp}$ long truncated duplication of atp 9 located on the minus strand together within $r n l$ in $A$. solipides and $A$. sinapina. We also found a 42 bp long duplication of atp 9 in $A$. borealis, which was located next to atp 9 after mobile element of the LAGLIDADG family.

Conclusion: Our study revealed active intronic and mobile genetic elements acquisition during evolution. Some introns have homology with rather distant species. We consider that horizontal DNA transfer has played a significant role in size variation and genome structure of the Armillaria mitochondrial genomes.

Acknowledgements: This study was funded by the Research Grant No. 14.Y26.31.0004 from the Government of the Russian Federation. 